Hansgerd Delbrück

\title{
Wolfskehls ironisches Verhältnis zur Mode
}

Es war Karl Wolfskehl keineswegs unwichtig, ob er den Zeitgeschmack der Mode traf oder nicht. Wer es gleichmütig ertragen soll, jenseits der Mode zu stehen, dem muß sie entweder gleichgültig sein oder er braucht ein starkes Selbstbewußtsein, wie es mit dem Hang zur Eitelkeit, der Wolfskehl eignete, nicht leicht vereinbar war. Wolfskehl versuchte früh, das Problem zu bewältigen, indem er die Mode, soweit das bei seinem Konservatismus überhaupt möglich war, an vorderster Front mitbestimmte: mit einem Wertekanon, der sich aus einer nach seiner Auffassung neu belebbaren und fortzuentwickelnden Vergangenheit speiste. Am erfolgreichsten war er mit diesem Ansatz zu Beginn des Jahrhunderts in der Szene der Münchener Bohème als vielgefeierter "König (oder Zeus) von Schwabing". Mit seiner Dichtung dagegen hatte er von Anfang an gegenüber der Mode einen schweren Stand, und die naheliegende Lösung, die fehlende Resonanz durch ironische Dis$\operatorname{tanz}$ nicht nur zur Mode, sondern auch zu sich selbst und zum eigenen Werk zu kompensieren, erschien ihm desto verlockender und zugleich problematischer, je offensichtlicher sich der Zeitgeschmack von seiner Dichtung entfernte.

Wolfskehl selbst scheint das bereits in einem Gelegenheitsgedicht von 1930 reflektiert zu haben:

Was von Tanten, ältern Schwestern

Lächerlich im Spinde hing -

Wahrt es treu! Das Modegestern

Schätzt kein Wissender gering.

Wespentaille, pralle Glieder, Hüften wie bei Grossmama -

Schon ist das verulkte Mieder

Samt dem Busen wieder da.

Gestern Taft und heute Kreppe,

Morgen wieder steifer Taft,

Kniefrei gestern, heut die Schleppe,

Gestern glitzglatt, heut gerafft. 
Freu dich, liebe alte Base,

Stelzend im verstaubten Chik:

Alles staunt, keins rümpft die Nase,

Wirkst als letzter Modetrick!!

Auf den ersten Blick scheint die Sympathie des Dichters hier auf seiten der alten Base zu liegen. Die Oberflächenironie der Verse wird von der ersten Strophe an dadurch erzielt, daß das Alte nicht nur trotz, sondern gerade wegen seiner Modefremdheit die Chance zu modischer Rehabilitierung bekommt. Ein unberechenbarer, aber doch jederzeit möglicher und, wenn man nur lange genug wartet, wahrscheinlicher Vorgang. Die Ermunterung der alten Base, sich über ihr plötzliches Comeback in der Mode zu freuen, ist indessen hinterhältig: sie wird aufgefordert, von einer Modetorheit zu profitieren, vor der sie ihr Alter bewahren müßte. Weil ihr einziger Beitrag zur neuen Mode im unbeirrbaren Festhalten an den einmal gewählten und von der Mode überholten Vorlieben besteht, identifiziert sich Wolfskehl mit der Base (wie mit der Mode) in der Form des ironischen Abstands. Damit verliert umgekehrt der für dieses Gedicht zentrale gnomische Satz aus der ersten Strophe, dem zufolge kein Wissender das "Modegestern" gering schätze, zuletzt seine ironische Relativierung und wird in prägnantem Sinne wahr. Nicht die Mode, für die unterschiedslos alles Alte eines Tages wieder modisch werden kann, sondern allein der Wissende weiß an dem Vergangenen das zu schätzen, was über die Vergangenheit hinaus Gültigkeit beanspruchen darf.

Für den bei der Niederschrift dieses Gedichts bereits einundsechzigjährigen Dichter hat das Gedicht offenbar apotropäische Funktion: Er wappnet sich gegen das Odium des Aus-der-Mode-Geratens mit der festen Absicht, sich durch das Modebarometer in keiner Richtung im eigenen Befinden beeinflussen zu lassen, weil das lächerlich wäre.

Eine Dekade später, nach der Erfahrung des Naziregimes und des doppelten Exils aus Deutschland und aus Europa, wird diese Absicht jedoch immer härter auf die Probe gestellt. Wie sehr, zeigt sich in seinem prekären Verhältnis zu dem ungleich erfolgreicheren Thomas Mann, dem im kalifornischen Exil lebenden weltberühmten Romancier, mit dessen Weltruhm er nicht mithalten kann. Am

1 Wolfskehl, Karl, Gesammelte Werke, hrsg. Margot Ruben und Claus Victor Bock, I (Dichtungen, Dramatische Dichtungen), Hamburg 1960, S. 250. (Im folgenden: GW I; auf diesen Band beziehen sich alle nur mit Seitenangabe belegten Zitate von Wolfskehls Gedichten.) 
6. März 1939, also nur ein halbes Jahr nach seiner Ankunft in Auckland, bedankt er sich in einem Brief an den Kollegen für das Empfehlungsschreiben, das dem aus Italien mit einem Besuchervisum nach Neuseeland gekommenen Wolfskehl bei den dortigen Einwanderungsbehörden den erwünschten, für ferneren Aufenthalt unabdingbaren Status eines "Permanent Resident" erwirken sollte. Der Dank klingt so, als wenn sich der Schreiber die gebotene Herzlichkeit mit einiger Mühe abfordern müßte. Angesichts der "in Betracht kommenden minds and mentalities", so formuliert er da, "nehme" er das von Thomas Mann aufgesetzte Gutachten "gern hin": "nicht nur um des Zweckes willen", sondern auch als "Freundesruf", der "in der Einsamkeit Antithules sonderlich gut" tue. Das mit solcher Schlußformel halbwegs überspielte Mißvergnügen an der generalisierenden Begründung des Gutachtens (Wolfskehl kennzeichnet es mit der Jean Paulschen Formel "mehr und dergleichen") dürfte in erster Linie dadurch hervorgerufen worden sein, daß in dem in unbeholfenem Englisch abgefaßten Schreiben seiner Lyrik gegenüber seiner Essayistik nur der zweite Platz eingeräumt schien. Thomas Mann hatte additiv2 formuliert, für Wolfskehl aber lag es nahe, die Aufzählung im Sinne eines hierarchisch gestuften Urteils zu verstehen und hieraus eine implizite Abwertung seiner Lyrik herauszulesen. Hatte doch Thomas Mann schon früher einmal, wie Wolfskehl ihn nun in seinem Dankesbrief erinnert, die Äußerungsformen der Lyrik "archaisch" genannt, weshalb der so gekennzeichnete Dichter sein Dichtertum nun ironisch mit einem aus der Antike genommenen Wort des römischen Senators Symmachus verteidigt: "daß es alten Leuten anstehe, bei ihrer gewohnten Daseinsform zu verbleiben." (BaN II, 293) Das Altersargument belegt erneut den schon beim obigen Gelegenheitsgedicht beobachteten zweisinnigen Gebrauch von Wolfskehls Ironie: was sich als sarkastische Selbstironie gibt, ist mindestens so sehr gegen den nicht einmal sechs Jahre jüngeren Thomas Mann gerichtet: Dessen Fähigkeit, den Zeitgeschmack zu treffen, erscheint dem Dichter nicht wirklich bewundernswert, sondern Beleg einer

2 "In his chief works Karl Wolfskehl deals with German and general European history of literature and culture, of religion, philosophy and esthetics. Besides his own poetry especially his translations of German and Latin medieval poems have won him a high reputation throughout the literary world, and so did the philosophical essays gathered in his book Bild and Gesetz (Image and Idea)." (Wolfskehl, Karl, Briefwechsel aus Neuseeland 1938-1948, hrsg. Cornelia Blasberg, Vorw. Paul Hoffmann, Darmstadt 1988, II, 1085 (Im folgenden: BaN II). 
Oberflächlichkeit, die er bei einem Menschen dieses Alters unnatürlich oder sogar geschmacklos findet.

Hierzu paßt genau, daß Wolfskehl es in einem Brief an Edith Landmann vom 4. 12. 1946 emphatisch ablehnt, mit einem Thomas Mann verwechselt zu werden. ${ }^{3}$ Gegenüber Thomas Mann selbst dagegen steigert er in einem Brief vom 12. 6. 1946 den Sarkasmus seiner Selbstironie: "Jenes von Ihnen einmal irgendwo unter die 'archaischen Künste' gezählte Laster des Versemachens übt der auch sonst Überalte heute noch." Und da er von Erich Kahler die Nachricht erhalten hatte, daß sich Thomas Mann einer Operation hatte unterziehen müssen, entbietet er seine Genesungswünsche "dem, von mir aus gesehen, .knapp der Jünglingsstufe Entwachsenen." (BaN II, 296) Hier spätestens hätte das Forcierte der selbstironischen Bescheidenheitsgeste dem Addressaten verständlich machen können, wie ungerecht behandelt sich Wolfskehl durch die Bezeichnung der Lyrik als einer "archaischen" Kunst tatsächlich fühlte - und daß sich hinter der Bescheidenheitsgeste ein Angriff auf Thomas Mann verbarg. Schwer zu begreifen und sicher nur mit Thomas Manns eigener Eitelkeit zu erklären ist deshalb, $\mathrm{da}$ der die gegen ihn gerichtete Ironie nicht bemerkt, sondern mit umgehender Post in stolzer epischer Detailfreudigkeit die Natur seiner überstandenen Krankheit erläutert, eingeschlossen "X-ray Bilder", Bronchoskopie und Namen des berühmten Lungenchirurgen, der ihm bei der Operation eine Rippe entfernt hatte, und sich zum Schluß dann allen Ernstes wegen seiner nun auch ärztlich attestierten Jugendfrische ein Selbstlob ausfspricht: "Die Ärzte versichern, ein Dreißigjähriger hätte nicht gelassener durch die Affaire hindurchgehen und weniger 'Geschichten' machen können. Ich muß sagen: Das gefällt mir an mir. Auch eine Rippe hat man dabei von mir genommen: Ist aber kein Evchen daraus geworden." Natürlich will Thomas Mann mit dieser Kadenz, entsprechend der gewöhnlichen rhetorischen Definition von Ironie, das fehlende Evchen gerade nicht als staunenswert, sondern als Beweis seiner zuletzt doch vorhandenen Einsicht in die Begrenztheit seiner Leistung als jugendlicher Patient verstanden wissen.

3 "Allgemeinheiten, zeitliche Überblicke, Kritik der Umstände und Geschehnisse sind nicht meine Art, nicht meines Fugs. Verwechseln Sie mich nicht mit Thomas Mann!" (BaN II, 817) Genauso deutlich in Briefen an Emil Preetorius vom 17. 3. 1947: ("[...] ich möchte nicht mit Thomas Mann verglichen werden, so wie Du's mit einem Seitenblick tust.") und vom 12. 6. 1948: "Ich stehe mit dieser Art Weltberühmtheiten, besonders der in question, in fast gar keinem Kontakt. [...] ehe ich von einem Thomas Mann einen Bettelscheck einlöse, hänge ich mich auf an geborgtem Strick." (BaN II, 949 und 960-961) 
Wolfskehl jedoch reagiert darauf mit dem Widmungsgedicht "Das Rippchen: für Thomas Mann", das er mit den Worten "Poeta Archaicus" unterzeichnet und Thomas Mann als Antwort auf seinen Brief zuschickt: eine kaum verhüllte Attacke auf Thomas Manns Defizit an Männlichkeit und also Denunzierung des Mannschen Werks als einer bloßen Eunuchenkunst. Das hat mit der Zahl der Lebensjahre nicht das Geringste mehr zu tun und auch nichts mit dem Gegensatz von archaisch und modern (oder in Wolfskehls Sprache "modisch"), sondern grenzt an Charakterdenunzierung:

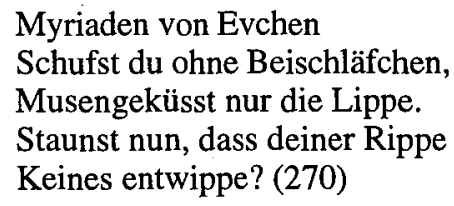

Die vom Scherzton kaum gemilderte Grobheit der Attacke bestätigt nicht nur Wolfskehls so ganz anderen Begriff von Selbstironie, der ihm jeden Sinn für Thomas Manns Selbstironie versperrte, sondern das Ausmaß des eigenen Leidens unter dem fehlenden Widerhall seiner Dichtung. Je mehr die Bedingungen seiner Exilexistenz und seine Altersbeschwerden sein Werk bedrohten, desto mehr war er darauf angewiesen, daß er sich der eigenen Vitalität in dem Bereich versicherte, der für ihn bis zuletzt der "eigentliche" 4 war: die deutsche Dichtung und sein eigenes Werk als Teil dieser Dichtung.

\section{2.}

Die Bedeutung von Wolfskehls Reflexion des Kriteriums der Mode für sein religiöses Spätwerk ergibt sich erneut aus dem Gedicht "Medico Magistrali" (268) von 1941. Während er jedoch in seinem Gedicht von 1930 dessen Heldin, die "liebe alte Base", durch die beigefügten Adjektive mit freundlichem Spott in ihrem unbekümmert einfältigen Verhältnis zur Mode gekennzeichnet hatte, ist die Mode diesmal über ihn selbst hinweggegangen, und er spricht über die eigene Zugehörigkeit zum "Modegestern" als ein

4 Paul Hoffmann berichtet in seinem Vorwort zum Briefwechsel aus Neuseeland 1938-1948 über Wolfskehls letztes Lebensjahr: "Auch auf dem Krankenlager sprach er am liebsten, wenn es die Schmerzen zuließen, und sich Gelegenheit bot, von dem, was er "das Eigentliche" nannte, was ihm seit je wesentlicher Lebensinhalt gewesen war: von deutscher Dichtung. Er überprüfte sein Spätwerk, noch immer auf Verbesserungen bedacht." (BaN II, 43) 
"Wissender", der sich über seinen Zustand keinerlei Illusionen macht. Im einzelnen hält er sich hier dùrchaus an das, was das ironische Rezept seines Gedichts von 1930 zum Umgang mit der Mode empfohlen hatte: er besinnt sich auf das bewährte Alte. Jedoch so, daß er, frei von jedem Schielen auf die Tagesmode, die Sprache des bewährten Alten gleichwohl in eine neue, unverkennbar Wolfskehlsche Sprache verwandelt:

\section{MEDICO MAGISTRALI}

Für Dr.W.G. in Dunedin, Neuseeland

Vom Fug des Schicksals darf ich nicht verlangen

Dass Jahre mir spurlos vorbeigegangen.

Ein jedes griff in meines Rades Speichen,

Schwang um, hielt auf, und hinterliess ein Zeichen.

Das eine gab mir einen Gallenstüber,

Das andre bog den Nacken langsam über,

Gar manches legte aufs Gesicht die Hand:

Innen wards licht, doch Tagesstrahl entschwand.

Das wieder fuhr nach Sohl- und Fussgelenken,

Schwer ward der Schritt, Fessel begann zu schwenken.

Dies nahm die Lust gewaltigem Schlurf und Mampfe,

Dies tat den Fingern weh im kralligen Krampfe.

Eins strich die Haare, dass sie graulich schüttern,

Und schob mich von den Töchtern zu den Müttern.

Verblasst der Glanz wie bei der Erbkommode -

Dübel spleisst, Schuber knarrt: bist aus der Mode!

Rage doch treibenden Safts, wenn Windsbraut nackt

Altstamm und Knorrgeist wild im Runde packt,

Und unsichtbar mein Vöglein, weil sie wettert,

Das Lied der Riesin siegreich überschmettert:

Sein Herz pocht machtvoll, ob es jauchzt, ob bangt,

Denn es ist keines, das im Nihil hangt.

Auckland 1941

Das Gedicht wurde von den Herausgebern unter die Rubrik "Gelegentliches" eingeordnet, und die Bezeichnung trifft zu. Es wäre aber ganz verkehrt, daraus auf Qualität zweiter Wahl, auf eine bloße Nebenarbeit zu schließen. In einem Brief an Edith Landmann vom 4. 12. 1946 hat Wolfskehl, bezogen dort auf sein Gedicht "An die Deutschen", unter Berufung auf Goethe dargelegt, welch hohe, ja höchste Meinung er vom Genre des Gelegenheitsgedichts hatte: 
"Aber das ist meine Art: ich spreche, ich denke, ich urteile von dem Winzigpunkt Ich aus. Auch dies Gedicht eines der 'Gelegenheit' in Goethes Verstand. Ob und wieweit es, grade durch diese Grenzziehung in Zeit und Ort [...] typische Gewalt erhält, Vor-Bild wird, habe ich nicht zu entscheiden." (BaN II, 817)5 Was also war die "Grenzziehung in Zeit und Ort" dieses Gedichts?

Auszugehen ist von der konkreten persönlichen Situation, in der das Gedicht geschrieben wurde. Mit Sicherheit war eine ernsthafte Krankheit im Spiel. Sie zwang ihn, im Umgang mit seinen Mitmenschen gegen seine Regel zu verstoßen, sich von den hiobhaft anwachsenden Gebrechen seines Alters keinesfalls in seiner umfangreichen Korrespondenz behindern zu lassen. Ein Jahr vor der Abfassung des Gedichts, in einem Brief an Wolfram von den Steinen vom 3. 4. 1940, hatte er noch schreiben können: "[...] zwar zerschleißt man in alten Tagen unvermeidlicherweise - ein Prozeß, der unter widrigen Umständen besonders unerbittlich sich vollstreckt - aber selbst sub specie dieser tristen Wahrheit wäre ein weiteres Schweigen Ihnen gegenüber gar zu schäbig." (BaN II, 307) Daß seine gesundheitliche Lage sich im Jahre 1941 erheblich verschlechterte, ergibt sich, wenn wir uns auf seine veröffentlichte Korrespondenz verlassen dürfen, aus der Lücke in der Reihe von ihm verfaßter Briefe. Sie reicht von Mitte August bis fast Ende November 1941. Gegenüber Verwandten und Freunden war er äußerst scheu und zurückhaltend in der Benennung seiner Krankheiten: in einem Brief vom 25. 11. 1941 an Pater Thomas Michels, offenbar schon während der Rekonvaleszenz; spielt er die hinter ihm liegende Krankheit herunter, indem er von ihr nur als von "allerlei äußere[n] Unebenheiten" spricht, die nunmehr "soweit geglättet" seien, daß er beginnen könne, aufgehäufte Briefschulden langsam abzutragen. (BaN II, 280) Und aus einem acht Tage später geschriebenen Brief an die Nichte Marie Luise Wolfskehl wissen wir nur, daß zu den "Unebenheiten", die ihn am Briefeschreiben hinderten, auch eine sich anschließende schwere Grippeerkrankung von Margot Ruben gehörte, 6 mit der er zusammenlebte, der er in

$5 \mathrm{Vgl}$. auch den früheren Brief vom 28. 2. 1940 an Curt von Faber du Faur: "[...] es gibt überhaupt keine äußern Umstände, nicht die Sachlage, sondern die Ichlage bestimmt Grad und Art des Lebensgefühls und sie, sie allein ist Quelle und Ziel, freilich nicht in irgendeinem monadischen Sinn." (BaN II, 77)

6 Wolfskehl begründet in diesem Brief vom 2. 12. 1941, warum sein Briefschreiben "im Spätsommer und Herbst" - also dem neuseeländischen Spätwinter und Frühling - immer mehr ein Problem geworden sei: Erst sei er selbst "eine Zeitlang unwohl" gewesen, und dann habe Margot Ruben eine sehr schwere 
jenen Jahren des Exils seine Briefe zu diktieren pflegte und ohne deren Hilfe er wegen der fortschreitenden Erblindung seine Korrespondenz nicht hätte führen können.

Worum es sich bei der eigenen Krankheit des Jahres 1941, die ihn ans Bett fesselte, 7 medizinisch tatsächlich handelte und wie schwer sie war, ergibt sich jedoch aus einem fünf Jahre später geschriebenen Brief Wolfskehls an Abraham Scholem Yahuda vom 2. 8. 1946: "Ich hatte damals einen so schweren Anfall von Herzmuskelschwäche, daß ich fast zwei Monate bettlägerte und seitdem, wie Faust von sich sagt, 'leise und bedächtig' die Schritte zu nehmen gezwungen bin." (BaN II, 270) Diese Krankheit sei für ihn "ein Erlebnis" gewesen: "eines von denen, die zur Bereitschaft mahnen." Keine Frage, daß er damit den Tod meint, denn Ausgangspunkt für seine Erwähnung des eigenen Herzanfalls gegenüber Yahuda war gewesen, daß auch dieser soeben von einer Herzattacke "angegriffen" worden war; Wolfskehl hatte auf diese Nachricht mit der Feststellung reagiert, wer "mal so um die 60 rum" sei, müsse "eigentlich immer gewärtig sein, daß einmal an die Tür geklopft wird." (Ebd.) Der Satz wirkt erneut wie eine Anspielung auf die Szene "Mitternacht", aus der schon das eben zitierte, von Wolfskehl explizitgemachte Faust-Zitat genommen war: die Szene, in der Faust erblindet und mit seinem Tod konfrontiert wird.

Davon, daß er selbst am Rande des Todes stehe, hatte Wolfskehl schon ein Jahr nach seiner schweren Erkrankung, am 25. 11. 1942, in einem Brief an Hanne Marcus gesprochen und sich auch da auf den Zustand seines Herzens bezogen. Doch hatte er da noch, obwohl er ausnahmsweise von "Leiblichem" zu reden bereit war, dieses Leibliche sogleich wieder mit Geistig-Seelischem vermischt: "Auch im Leiblichen bin ich nicht mehr, der ich war. Ich schwanke. Mein Herz will nicht mehr. Müde und verscheucht. Ich will Dir nichts vorklagen." (BaN II, 410) Im Geistigen ist die Folge seiner Krankheit eine Resignation, die er positiv zu fassen sucht: "Ich verstehe jetzt so vieles. Immer mehr und mehr sehe ich, wie alles in und außer dem Fug dennoch den Weg bereitet hat. Ich

Lungenentzündung bekommen: "man konnte feststellen, wie auch das engste Lebenssystem durch den Zusammengriff von Räderchen in Gang gehalten wird, bei denen keins ausfallen darf." (BaN II, 496) So sehr er also seine eigene Krankheit herunterzuspielen suchte, so unumwunden war er bereit, die Krankheit Margot Rubens beim Namen zu nennen.

7 Vgl. den Brief an Schocken vom 12. August 1941: "Auch meine Gesundheit ist in letzter Zeit nicht recht fest, augenblicklich hüte ich das Bett." (BaN II, 204) $\mathrm{Da} \beta$ er das Bett hüten muß, ist der erste Hinweis darauf, daß es sich um eine ernstere Krankheit handelt. 
finde mich $a b$, ob ich fand oder nicht, ich finde mich ab." Trotz seines Versuchs einer positiven Resignation war er beim Schreiben dieses Briefes ganz offensichtlich an einem seelischen Tiefpunkt angelangt, wie er ihn seinen Briefen nur selten anvertraut hat: "Der Schleier, den das Leben webt, Du sprichst von ihm, Du webst noch an ihm, mir gleitet er aus den Händen, und die bannenden Worte, ärmer werden sie, matter."

Doch wie sein Spätwerk überhaupt, ist gerade auch "Medico Magistrali" Beweis für seine Fähigkeit, die mit der physischen Schwächung des Herzens einhergehende Müdigkeit der Seele mit immer erneuter Bannkraft8 seines Worts zu überwinden. Im Falle von "Medico Magistrali" war dabei offensichtlich seine Beziehung zu dem Arzt "Dr. W. G." beteiligt, dem er das Gedicht gewidmet hat. Von diesem Arzt hier nur so viel: Es handelte sich um den im Süden der Südinsel - man denke an die mehr als doppelte Entfernung Hamburg-München -, in der Universitätsstadt Dunedin forschenden und mit Lehraufträgen betrauten deutschen Emigranten Dr. Walther Griesbach, ehemals Professor in Hamburg. Wolfskehl hatte ihn im Frühjahr 1941 (also im neuseeländischen Herbst vor dem Winter, in dem er dann an seiner Herzschwäche erkrankte) auf einer Reise auf die Südinsel kennengelernt, und sein Vertrauen, Dr. Griesbach teile sein Mediziner-Ideal, speiste sich aus dem Bewußtsein der gemeinsamen humanistischen Vorbildung. Die Vermutung liegt nahe, daß Wolfskehl in ihm einen späten Kollegen Fausts sah, der schon bei Goethe zugleich Arzt und Universitätslehrer gewesen war, und dies in vielfachem Konflikt mit den Autoritäten. Griesbachs deutsche Examen waren in Neuseeland nicht anerkannt, weshalb er dort einerseits nicht praktizieren, andererseits aber an der Universität von Dunedin forschen und lehren und auf diese Weise durch sein Fachwissen zur Wohlfahrt des Landes beitragen durfte. So meint das Gedicht im Dativ seines Titels "medico magistrali" wohl nicht nur den "meisterlichen" Arzt, sondern ist zugleich Anspielung auf eine weitere in der Medizinersprache übliche Verwendung des Adjektivs "magistralis", das - als Gegensatz zu "officinalis" - nicht in das amtliche Verzeichnis auf-

8 Natürlich meint Wolfskehl mit den "bannenden" Worten in seinem Brief an Hanne Marcus etwas ganz anderes, als daß sie den Leser in Bann schlagen. In dieser letzteren, flachen Bedeutung benutzt Wolfram von den Steinen den Begriff in einem Brief vom 18. Januar 1931, indem er sagt, er bewundere "vor allem Ihre Kraft mit erstem Wort schon zu bannen und dann den Leser ebenso liebenswürdig wie unausweichlich bis zum letzten Wort an Ihrer Gedankenführung festzuhalten." (BaN II, 123) 
genommene, also nicht amtlich zugelassene medizinische Rezepte bezeichnet, deren Wert gleichwohl unbestritten ist. Jedenfalls ist das Gedicht kaum denkbar ohne Wolfskehls doppelte Fähigkeit: in dem anderthalbtausend Kilometer entfernten Griesbach eine Reinkarnation des Arztes und Universitätslehrers Faust $\mathrm{zu}$ sehen und sich zugleich auch selbst mit Faust als dem alternden, erblindenden und mit seinem Tod konfrontierten Menschen zu identifizieren. Erst die literarische Überhöhung läßt den Widmungsaddressaten, der bei Wolfskehls Erkrankungen von Dunedin aus um telefonische Fernbehandlung gebeten $\mathrm{zu}$ werden pflegte, als Initiator der vom Gedicht nicht nur reflektierten, sondern geleisteten Selbstheilung plausibel werden. Daraus ergibt sich umgekehrt, daß die "typische Gewalt", die diesem Gedicht nach der von Wolfskehl gegenüber Edith Landmann gebrauchten Formel aus der Selbstheilung zuwächst, auch den Arzt und Lehrer Griesbach zuletzt wieder in die Rolle des vom "Vor-Bild" Lernenden verweist: nicht anders als den Dichter und alle seine Leser. In Wolfskehls humanistischer Sicht war die Rolle des Lehrenden von der des Lernenden grundsätzlich nicht zu trennen.

\section{3.}

Von hier aus erklärt sich Wolfskehls Entscheidung für die äußere Form seines Gedichts: die uns von Fausts Monologen in Faust I vertrauten Madrigalverse. Die durchgängig paarweise gereimten Verse, die im Druckbild ohne jede strophische Gliederung auskommen, können nur oberflächlicher Betrachtung einsinnig-monoton erscheinen. Dem genauen Hinsehen und vor allem Hinhören, das Wolfskehls Dichtung einzig angemessen ist, ergibt sich die strophische Gliederung (in vier Quartette und ein Sextett), bietet sich eine vielfältig gespannte rhythmische Struktur und erschließt sich die Vielsinnigkeit9 der Komposition. In ihr mischen sich - "im Ganzen wie im Einzelnen", also von der ersten bis zur letzten Zeile - Autobiographisch-Privates und Öffentlich-Politisches, Physiologisches und Geistig-Literarisches, Irdisches und Metaphysisches. Dabei spricht das Ich in "Medico Magistrali", wie bereits ange-

9 Als "vielsinnig" hat Wolfskehl selbst den Stil seiner späten Dichtung in einem Brief an Abraham Scholem Yahuda vom 2. 8. 46 gekennzeichnet: ihre "Komposition" sei "altersgemäß so dunkel und vielsinnig, daß sich bei Lebzeiten mindestens wohl kein Verleger dranwagt." (BaN II, 271) Das war auf den Zyklus Hiob oder die Vier Spiegel bezogen, gilt aber offenbar genauso für das Gedicht "Medico Magistrali." 
deutet, keineswegs nur die Sprache des Goetheschen Faust: das widerspräche Wolfskehls wie Goethes Vorstellung von der Verpflichtung des Ich zu individueller Eigenart.10 Gleich die geballte Wortfolge des Anfangs ("Vom Fug des Schicksals") ist weder Faustisch noch Goethisch, vielmehr ganz Wolfskehlisch. Das Wort "Fug", von Wolfskehl besonders häufig auf jüdisches Schicksal angewendet, markiert mit dem Fug und Recht des Schicksals zugleich dessen Unerbittlichkeit: das Verletzende, das mit dem vom allgemeinen Sprachgebrauch zu erwartenden Wort "Fügung" unzulässig geglättet wäre.11 Der Genetiv "Schicksals" ist nicht jambisch, sondern spondäisch mit zwei Hebungen zu lesen, wodurch der Vers in der Mitte eine Zäsur erhält, die der vom Fug des Schicksals provozierten Nachdenklichkeit, als einem Einhalten des gewöhnlichen Denkens, gemäß ist. Eine ähnliche Korrektur oberflächlichen Denkens findet sich in der rhythmisch-syntaktischen Abfolge des zweiten Verspaars. Die Schicksalsschläge, denen das Ich im ersten Vers Jahr für Jahr auf scheinbar immer gleiche Weise ausgesetzt ist, werden in ihrer Abfolge im zweiten Vers rhythmisch unterbrochen: gleich anfangs beschleunigt, dann zweimal aufgehalten und zuletzt wieder zu einförmig syntaktisch-rhythmischem Fließen gebracht:

\section{Ein jedes griff in meines Rades Speichen,}

Schwang um, hielt auf, und hinterließ ein Zeichen.

Das Zeichen, mit dem das Ich über die Jahre in seinen Krankheiten und Gebrechen immer wieder neu und anders und doch auch wieder einförmig-einsinnig "gezeichnet" ist, gibt den ersten vier Versen, die als in sich geschlossene Einleitung des Gedichts anzu-

$10 \mathrm{Vgl}$. seine Kritik an den Gedichten von Julius Schwabe im Brief vom 18. Dezember 1929: "Kein eigener Blick, kein eigner Ton." (BuA, 46) Die Eigenheit des Tons unterscheidet das Gedicht "Medico Magistrali" auch deutlich von Wolfskehls eigenem Verfahren in Maskenzug 1904, wo er in der Maske des Gianino ganz bewußt und so rein wie möglich die Sprache Hofmannsthals aus Der Tod des Tizian hatte imitieren wollen. (In Maskenzug 1904 wirkt das Gelungene der Imitation heute leicht wie Parodie, obwohl es mit Sicherheit nicht so gemeint war.)

11 Dies scheint ein eminent jüdischer Zugang zum Begriff des Schicksals. So verwundert es nicht, daß sich bei Elazar Benyoëtz (Variationen über ein verlorenes Thema, München 1997, 128) Gertrud Kantorowicz' Satz zitiert findet, unser "ganzes Leben" werde "nur gewonnen, wo wir auch das noch aufnehmen, was uns vernichtet." Indessen stammt dieser Satz, wie von Benyoëtz in den Zitatnachweisen gewürdigt, aus ihrem Buch Vom Wesen der griechischen Kunst, hrsg. Michael Landmann, Heidelberg/Damstadt 1961, 78. 
sehen sind, einen gewichtigen, metaphysisch getönten Abschluß. In ihm bekennt sich das Ich gleich eingangs zu seiner jüdischen Schicksalsperspektive, wie Wolfskehl dies zur Zeit seiner Emigration auch brieflich zu tun pflegte.12 Entsprechend weist die Aufzählung und Beschreibung der Krankheiten und Gebrechen in dem dann folgenden Hauptteil, also in den nächsten dreimal vier Versen, von vornherein über sich hinaus. Die Leiden haben, bei all ihrer realen Schwere und Überschwere, einen Sinn, der sich nicht in ihnen selbst erschöpft; ihn $\mathrm{zu}$ belegen und $\mathrm{zu}$ bewähren ist die von Anfang an angestrebte Aufgabe, die dann zuletzt in den Schlußzeilen des Gedichts erreicht wird.

Im ersten Verspaar des zweiten Quartetts wird jedoch, zur Kennzeichnung der Vielfalt und Verschiedenartigkeit der physischen Leiden, das rhythmische Tempo der metrisch gleich gebauten, in sich selbst nicht unterbrochenen Verse durch den Inhalt und die Lautfolge erst stark beschleunigt, dann stark verlangsamt. Der Plötzlichkeit und kurzen Dauer der Attacke einer Gallenkolik13 steht der schleichende Vorgang der durch Osteoporose hervorgerufenen Versteifung der Wirbelsäule14 gegenüber:

Das eine gab mir einen Gallenstüber,

Das andre bog den Nacken langsam über,

sowie gleich anschließend, nur durch Komma und Versende abgetrennt, weil dieses andere, sich über viele Jahre hinziehende, schleichende Übel das erste im Bewußtsein des Dichters überdeckt:

12 Vgl. im Brief an Edgar Salin vom 19. 1. 1947: "Das Schicksal kam über mich, ich hatte es nicht bloß zu ertragen, ich hatte es zu gestalten. Ich hatte die Zeichen $\mathrm{zu}$ deuten, ich hatte es als Wink zu verstehen. Und als Ruf hatte ich ihm zu folgen, dem Urväter-Fatum. All dies unterliegt keiner menschlichen Beurteilung, sondern dem höchsten Fug." (BaN II, 193)

13 Von einer Magen- und Gallenerkrankung berichet Wolfskehl z. B. in einem Brief an Salin vom 26. 7. 1938, sowie erneut (und diesmal mit dem Eingeständnis, daß es sich um eine viel schwerere Krankheit handelte, als im ersten Brief zugegeben) am 1. 11. 1938. (BaN II, 116 und 121)

14 Paul Hoffmann betonte gegenüber dem Verf. in einem Telefongespräch vom 27. 12. 1997 Wolfskehls auffällig aufrechte Körperhaltung. Sie steht indessen seinem Bewußtsein des gebogenen Nackens nicht entgegen, sondern zeigt umgekehrt, mit welch bewußter Anstrengung er an seiner aufrechten Haltung arbeitete. Im geistig-seelischen Bereich wird Gleiches schon durch eine Briefstelle vom 5. 6. 1939 (an Edgar Salin) belegt: "Dieser furchtbarste Verzicht, damals [sc. in Rom zur Zeit des Tacitus] schon erdämmernd in den Seelen und Stoa genannt, welche Würde trug er doch in sich! Denken wir daran, stehen auch wir aufrecht, ob auch verhüllt an Haupt, Sinn, Mutung und Wort." (BaN II, 126) 
der Vorgang des Erblindens. Die Jahre der Erblindung werden hier in biblisch verständlicher Allegorik im Akt des feierlichen Handauflegens vorgestellt; mit dem gespendeten Segen wachsender Erkenntniskraft verhängen sie zugleich den Fluch der Blindheit:

Gar manches legte aufs Gesicht die Hand:

Innen wards licht, doch Tagesstrahl entschwand.

Als Geste des gespendeten Segens wäre eher ein Handauflegen "auf's Haupt" zu erwarten gewesen. Die aufs "Gesicht" im engeren Sinne (also auf die Augen) gelegte Hand zeigt jedoch an, daß mit der Erblindung, wie in üblicher Metaphorik mit dem "Ende des Tages", bereits das Ende des Lebens selbst vorgestellt wird, an dem den Menschen die Augen für immer geschlossen werden. Das verweist hier zum erstenmal auch inhaltlich auf den Goetheschen Faust, und zwar auf den erblindenden Faust vom Ende des Zweiten Teils. Der wird, in der Szene "Mitternacht", von der Sorge sogleich mit völliger Blindheit angehaucht, und dieser Anhauch trägt für Faust, wie sonst der Anhauch der Pest, bereits den Tod in sich. Faust deutet den Vorgang verharmlosend als sich verfinsternde Nacht:

Die Nacht scheint tiefer tief hereinzudringen,

Allein im Innern leuchtet helles Licht. (V. 11499-11500)

In seiner Blindheit kann er den Tod weder "sehen" noch "hören" und deutet er das von den Lemuren bereits besorgte Graben seines Grabs in das Graben seines großangelegten Bewässerungsprojekts um. Das Ich des Gedichts kommt dagegen, in Identifizierung nicht mit dem Bewußtsein, sondern der objektiven Erfahrung des alten Faust, zu einem umgekehrten Fehlschluß. Es sieht sich bereits mit Blindheit und Tod konfrontiert, wo von ihm Weiterleben in der Erblindung gefordert ist. Diese Umkehrung drückt sich auch formal darin aus, daß die beiden Hälften des Verses, in dem das Handauflegen als Ursache der den Tod vorwegnehmenden Erblindung erscheint, wie ein spiegelverkehrt angeordnetes Zitat der beiden Faust-Verse wirken. Dabei beruht der Fehlschluß in beiden Fällen auf der Überschätzung der trotz äußerer Erblindung behaupteten inneren Lichtwerdung. Deren Grenzen zeigen sich genauso in der Voreiligkeit, mit der das Ich des Gedichts das noch zu bewältigende Lebensende mit der Vorwegnahme des Todes überspringt, wie 
umgekehrt in der Langsamkeit, mit welcher der nach eigner Berechnung "weise" gewordene Faust (V.' 11440) sein Leben von nun an, auch ohne das bewußt so zu reflektieren, ins Unabsehbare dehnen will, jedenfalls von jedem Denken an den eigenen Tod denkbar weit entfernt ist.

Anders als dem in der Erblindung todgeweihten Faust ist dem Ich des Gedichts, zugleich mit der belastenden Forderung des Weiterlebens auf dieser Erde, die positive Möglichkeit des Neueinsatzes geblieben, die wieder und wieder auch das Leben Fausts kennzeichnet, bis sie ihm erst ganz zuletzt abgschnitten wird. Im Gedicht mündet der gedanklich übereilte, aber seelisch verständliche Vorstoß von der zunehmenden Erblindung zu Blindheit und Tod in einer bedrohlichen Fermate, der nur durch einen Neuanfang zu begegnen ist. Folgerichtig wirkt in den nächsten vier Versen das Zurück ins Aufzählen weiterer (nicht passiv erlittener, sondern standhaft ertragener) Krankheiten wie eine bewußt gewählte Therapie; sie erlaubt es dem Ich, seine Lebensgeister fast routinemäßig wiederzubeleben und sogar, wenn auch nur kurzfristig, zu unerwartet schnellem Puls anzustacheln. Nachdem im ersten Vers die Symptome stechender Gicht- und Rheumaschmerzen,15 in Übereinstimmung mit ihrer Erscheinungsform, in schnellem Tempo benannt sind:

Dies wieder fuhr nach Sohl- und Fussgelenken,

fällt der zweite Vers, entsprechend den die Bewegungsfähigkeit behindernden Folgeerscheinungen von Gicht und Rheuma, sogleich zurück in einen schleppenden Gang:

Schwer ward der Schritt, Fessel begann zu schwenken.

Von der Verlangsamung der Gehfähigkeit kommt das Ich sodann auf das Schwinden eines früher unverhohlen ausladend genossenen, barocken Appetits, 16 und dazu kommen, ohne daß das aus der

15 Schon nach nur einem Jahr Exil in Auckland schreibt Wolfskehl am 23. 7. 1939 (neuseeländischer Winter!) an Curt von Faber du Faur: "Lieber Freund, der Winter ist hier im Lande der Wohnkisten und Feuerplätzchen eine klamme Angelegenheit rheumatischer Komplexion. [...] und denken Sie sich unterm Worte 'Winter' noch ein bißchen mehr als das bloß Jahreszeitliche. Ja, dieses als bloßes Symbol eines menschlichen Zustands, wie er - schwer halt ich C. G. Maassens Lieblingswort zurück - graulicher schwer vorzustellen ist. Ob ich damit nur die Meinung eines älteren Herrn mit gelegentlichem Gicht-Zuck in Hand oder Knie zum Ausdruck bringe? (BaN II, 72-73) 
Folge der Verse als Folgeerscheinung der früheren Eßlust abzuleiten wäre, die Schmerzen verkrampfter Finger als Schmerzen eigenen Rechts:

Dies nahm die Lust gewaltigem Schlurf und Mampfe, Dies tat den Fingern weh im kralligen Krampfe.

Bis zu dieser Stelle der Liste hat der Dichter die Jahre, denen er seine Gebrechen zu danken hat, mit "das eine" und "das andre" und dann zweimal mit "dies" benannt, sowie zwischendrin einzelne Jahre summarisch gebündelt in dem Wort "gar manches" zusammengefaßt. $\mathrm{Zu}$ Beginn der nächsten vier Verse kommt er jedoch auf das eine Jahr zu sprechen, das ihm unter allen herausragt, weil es ihm offenbar unter allen seinen altersbedingten Gebrechen das zuletzt doch am schwersten zu Ertragende gebracht hat, schwerer noch als die Erblindung: den Verlust der Jugend. Zum zweiten Mal ist hier die Identifizierung mit Faust unverkennbar:

Eins strich die Haare, dass sie graulich schüttern, und schob mich von den Töchtern zu den Müttern.

Die schütteren Haare erfüllen, in Verbindung mit der "Sohle" im ersten Vers des Hauptteils, das Schema des "von seiner Fußsohle bis zu seinem Scheitel" mit Krankheit geschlagenen Hiob. (Hiob 2,7) Darüber hinaus weisen die graulich schütter gewordenen Haare bereits voraus auf das Simson-Thema der in den Haaren versammelten und ohne sie verlorenen Manneskraft, das auch im Dritten Spiegel von Wolfskehls Hiob, in Hiob-Simson, eine Rolle spielt. Drittens schließlich ist gewiß keine Frage, daß der Dichter seine "graulich" schütternden Haare mit dem "graulichen" Zustand der Menschheit im Gefolge der von Deutschland ausgehenden Nazigreuel in Verbindung brachte.17 Auf die letzteren wird mit Be-

16 Im Jahre 1931, also zehn Jahre vor der schweren Herzerkrankung, hatte ihn eine schwere Darmerkrankung - oder wie er sich in einem Brief an Hans Schiebelhuth vom 8. 6. 1931 ausdrückt - "eine scheußliche Darmgeschichte" bereits einmal an den Rand des Todes gebracht: "Du weißt doch, wie krank ich war? 'Nie noch war der Tod ihm so nah geschritten' - heißts in meinem 'Saul'. So war es wirklich, ganz ungelogen." (BuA, 133) Und schon damals hatte Wolfskehl in der Krankheit eine Herausforderung an "Geist und Wille" gesehen. Während jedoch an dieser Stelle des Gedichts noch allein vom geschwundenen Appetit sowie von den gicht- oder rheumageplagten Fingern die Rede ist, hat Wolfskehl die Auseinandersetzung mit der Wirkung des Alterns auf Geist und Seele für die Klimax des Schlusses aufbewahrt.

17 In dem oben zitierten Brief an Faber du Faur, in dem Wolfskehl den Winter als 
zug auf das Ende des Gedichts zurückzukommen sein. Doch ist es zunächst das Problem der geschwundenen jugendlichen Liebesfähigkeit, das hier für Wolfskehls neues Faust-Zitat verantwortlich ist. Während das erste Faust-Zitat das Bild des steinalten, kurz vor seinem Tode blind gewordenen Faust aufrief, bezieht sich das zweite auf den Beginn des Zweiten Teils: Fausts Gang zu den Müttern aus der Perspektive des Verlusts der Liebe zu Gretchen gesehen: nicht als Gang zu dem Ursprünglichen und Eigentlichen, sondern als traurigerweise allein übrig bleibende Ersatzhandlung.

Das Thema der altersbedingt schwindenden Sexualkraft weist dabei sogar noch weiter zurück auf die Szene "Hexenküche" im Ersten Teil, in der Faust nicht nur im Hexenspiegel zum erstenmal das Bild der Helena gesehen, sondern auch die Jugendkraft erworben hatte, die ihn zu seiner Liebe zu Gretchen befähigte. In Wolfskehls Gedicht wird nun aber das Thema sogleich erweitert zum umfassenderen Problem des Nicht-mehr-Dazugehörens in einem offenbar sehr viel allgemeineren Sinne, der auch und vor allem sein Dichtertum einschließt, und dies ist die Stelle, an der er sein Problem ausdrücklich als eines der Mode anspricht:

Verblasst der Glanz wie bei der Erbkommode -

Dübel spleisst, Schuber knarrt: bist aus der Mode!

Die Bitterkeit in der Ironie dieser Verse zeigt bereits an, daß Wolfskehl hier auf Umwegen wieder zu der Todesthematik zurückgefunden hat, die er - mit dem Verzicht auf den am Ende des zweiten Quartetts erreichten tragischen Ton - zunächst so erfolgreich hinter sich gelassen hatte. Dabei scheint der Selbstvergleich des Ich mit einer Erbkommode durch jenen Sarg angeregt, der zu Beginn von Gottfried Kellers Roman Der grüne Heinrich (Zweite Fassung) dem über den Tod reflektierenden Titelhelden das ist, was Faust das Grab.18 Das tertium comparationis von Wolfskehls dich-

\footnotetext{
"Symbol eines menschlichen Zustands" deutet, wie er "graulicher schwer vorzustellen ist", hatte er die Frage, ob das vielleicht nur die Meinung eines "älteren Herrn mit gelegentlichem Gicht-Zuck" sei, als unerfüllbaren Wunsch verneint: "Fast möchte ich's wünschen, um des lieben Gottes willen noch mehr als wegen seiner Schutzbefohlenen, aber die Briefe, die man so aus aller Welt erhält, sind auf den gleichen Ton gestimmt, und so muß man sich denn bescheiden und im einzelnen $\mathrm{zu}$ erhalten und $\mathrm{zu}$ tun suchen, was einem noch obliegt sub signo jenes berühmten 'Als Ob'." (BaN II, 73)

18 Die zweite Fassung des Grünen Heinrich als Kellers epische Antwort der späten siebziger Jahre des 19. Jahrhunderts auf Goethes dramatisches Faust-Gedicht zu lesen hat einen eigenen Reiz, wie hier natürlich nur ausschnitthaft deutlich
} 
terischem Selbstvergleich mit der Erbkommode als Sarg ergibt sich, wenn wir nicht nur auf des Möbels konstituierende hölzerne Bestandteile (bei der Erbkommode Dübel und Schubladen, im Falle des Sargs die vom grünen Heinrich, dem Modetoren, synekdochisch genannten "vier Tannenbretter") abheben, sondern darüber hinaus, wiederum dem grünen Heinrich folgend und zugleich auf die Schlußverse des Gedichts vorausgreifend, das Möbelstück als Spätform des Baumes ansehen. Der grüne Heinrich reflektiert über den Baum sogleich in dessen Spätestform des völligen Verrottens seiner "vier Tannenbretter", in der er zuletzt von dem in völlige Verwesung übergangenen Menschen nicht mehr zu unterscheiden ist. Sub specie aeternitatis erscheinen dem grünen Heinrich Mensch und Baum deshalb von vornherein verwandten Geschlechts, und das Bild, in dem er sie als menschliche Leiche und als die besagten vier Tannenbretter gemeinsam unter die Erde kommen sieht, hilft ihm in der Verallgemeinerung des allen Menschen wie aller lebendigen Kreatur verhängten Endes, den bedrohlichen Gedanken an den eigenen Tod als eine sein jetziges Lebensstadium noch nicht betreffende Wirklichkeit ebenso aus dem Bewußtsein zu verdrängen, 19 wie dies Faust mit der Umdeutung des Grabgeräusches der Lemuren gelingt. Das Ich des Gedichts "Medico Magistrali" dagegen, das zunächst in seiner Identifizierung mit dem an

werden kann.

19 Der junge Heinrich beruhigt sich gleich zu Beginn des Romans in schöner Selbstüberschätzung über den Tod als über eine naturnotwendige Voraussetzung des Lebens, da, wie er in seiner naturwissenschaftlich aufbereiteten Privattheologie vor sich hin philosophiert, die Graberde des Gottesackers seines Heimatdorfs schon längst nicht nur "buchstäblich aus den aufgelösten Gebeinen der vorübergegangenen Geschlechter" besteht, sondern auch aus den "vier Tannenbretter[n], welche jedesmal mit in die Erde kommen und den ebenso alten Riesengeschlechtern auf den grünen Bergen rings entstammen." Bequemerweise übergeht er in seinem Vergleich der Menschen mit den Bäumen das Stadium, in welchem der in Bretter zerschnittene Baum von menschlicher Handwerkskunst zunächst einmal in ein möglichst haltbares Möbel verarbeitet ist, und sieht statt dessen den Sarg sogleich wieder in die "vier Tannenbretter" zerfallen. (Keller, Gottfried, Der grüne Heinrich, 86.-90. Aufl., Stuttgart, Berlin 1917, S. 11-12) Dieser Bequemlichkeit entspricht, daß er wenig später in der Erinnerung an den Vater dessen physischem Tod mit euphemistischer Metaphorik überspielt: "Er [sc. der Vater] ist vor der Mittagshöhe seines Lebens zurückgetreten in das unerforschliche All und hat die überkommene goldene Lebensschnur, deren Anfang niemand kennt, in meinen schwachen Händen zurückgelassen..." Übrig ist dem Sohn nur noch das "Bild", das er sich von seinem Vater, oder genauer von dem "Verhältnis zwischen einem Vater und seinem erwachsenen Sohne", "ausgemalt" hat, und selbst dieses Bild ist ihm in Wahrheit bereits "ein Teil des großen Unendlichen geworden", "unter dessen Obhut ich zu wandeln glaube." (30-31) 
seinem Lebensende angelangten Faust dem eigenen Ende ebenso vorausgeeilt war wie der grüne Heinrich mit der gedanklichen Gleichsetzung von individuellem eigenen und allgemeinem Tod, distanziert sich hier von dem allzu optimistischen Verfahren, mit dem der grüne Heinrich den menschlichen Verfallsprozeß erst mit dem physischen Tode beginnen sieht. Wie für einen jungen Menschen naheliegend, stellt der grüne Heinrich seine von der Erinnerung an den Tod des Vaters ausgelösten Reflexionen aus der Perspektive des überlebenden Nachfahren an. Der Selbstvergleich mit der Erbkommode zeigt das Ich des Gedichts dagegen im Stadium des Alters, in dem es noch selbst erleben muß, daß sein Wert von den Erben, wie Wolfskehl sich an anderer Stelle20 über mangelnde Wertschätzung von seiten seiner europäischen Verleger beklagt, nur noch "per dignitatem", also sozusagen idealiter, gewürdigt wird, während ihnen das Erbstück praktisch im Wege ist.

Dabei braucht das Ich, das sich im Gedicht mit einer Erbkommode vergleicht, keine Dritten, um sich von ihnen vorrechnen zu lassen, warum es für sie seinen "Glanz" verloren hat. Vielmehr hat das Möbel von dem Baum, aus dem es gemacht ist, noch genug Leben in sich, um das dichterische Ich selbst anreden zu können: "Bist aus der Mode." Als Stimmwerkzeuge dienen dabei die Einzelteile, die das Möbelstück als solches zusammenhalten und mit ihrem Funktionieren seinen Nutzen gewährleisten müßten. Nur ist es in diesem Fall gerade ihr Nicht- oder Falschfunktionieren, das hier, anders als das angenehm-stumme Verrotten des Sargs in der Vorstellung des grünen Heinrich, mißtönig laut wird: "Dübel spleisst, Schuber knarrt." Das Möbelstück Kommode, zu Beginn des 18. Jahrhunderts in Frankreich zur Förderung menschlicher (wie der Name sagt) Bequemlichkeit erfunden, wird seinen Besitzern unbequem, wenn die Schubladen klemmen, weil der spleißende Dübel die Konstruktion aus Lot und Fugen bringt: unbequem nicht nur für den Gebrauch, sondern auch dem Ohr. Da das Möbelstück im Gedicht jedoch nur dem Ich zum Selbstvergleich dient und wir es nur indirekt, eben durch die Stimme des Ich vernehmen, ist in ihr natürlich vor allem die eigene Verstimmung über sein Versagen vor der Mode eingefangen.

Gerade die Bewußtwerdung der Gefahr des modebedingten Tods seiner Dichtung, die den Dichter folgerichtig zum Gedanken an seinen bevorstehenden physischem Tod zurückführt, führt aber zum letzten, dramatischen Neuanfang und Aufschwung der letzten

20 Brief an Emil Preetorius von Ende Dezember 1947, BaN II, 957. 
sechs Verse. In ihnen wird die Entstehungsfolge von Baum und Möbelstück gleichsam um- und zurückgedreht in einer dichterischen Freiheit, die das Ich beschwörend-trotzig seiner Physis abverlangt:

Rage doch treibenden Safts, wenn Windsbraut nackt Altstamm und Knorrgeist wild im Runde packt [...]

Wolfskehl liebte es auch sonst, die sprichwörtliche Bezeichnung "von Saft und Kraft" in das Bild des safttreibenden Baums oder verwandte Bilder umzusetzen,21 und er liebte es, sich selbst mit einem Baum zu vergleichen.22 Entsprechend zielt er hier auf Umwertung des eigene Aitseins ins Positive des Altbewährten, Urwüchsigen, Standhaften, und wie die Befehlsform ("Rage doch") identisch ist mit der finalen Verbform der 1. Person des Indikativs Praesens, gerät die Selbstbeschwörung selbst zu Ausführung und Erfüllung. Erreicht wird sie hier durch eine dritte Identifizierung mit Faust, und zwar diesmal dem Faust der Walpurgisnacht mit ihrer Thematisierung von Sexualität, Alter und Mode.

Im Sturm der Walpurgisnacht "knarren" die Wurzeln der Bäume wie in Wolfskehls Gedicht die Schublade der Erbkommode, und ganze Bäume "splittern", wo sie im Gedicht zum Dübel verarbeitet folgerichtig nur "spleissen". Faust wird vom Sturm, der in der Walpurgisnacht die Baumstämme im "fürchterlich verworrenen Falle / Übereinander krachen" läßt, nicht umgeworfen; er ist nicht alt wie die anderen Alten (General, Minister, Parvenu, Autor), die in ihrer wehleidigen Klage über die verlorene Jugend und den Verlust des früheren Einflusses an das Problem erinnern, dem das Ich in Wolfskehls Gedicht mit der höhnischen Feststellung konfrontiert ist, "aus der Mode" zu sein. Während Mephisto ihrer aller dahingeschwundenen Einfluß als bloße Folge altersbedingter Sexualschwäche erscheinen lassen will ("Und weil mein Fäßchen trübe läuft, I So ist die Welt auch auf der Neige"), erfährt Faust vom Sturm ausdrücklich Belebung:

Wie rast die Windsbraut durch die Luft!

21 In einem Brief an Emil Preetorius vom 23. 5. 1930 rühmt er Yahuda mit den Worten: "Gottlob es gibt noch Saftwuchs in der Welt!" (BuA, 81)

22 Vgl. z. B. im Brief an Preetorius vom 23. 1. 1948: "[...] die Spätphase im Exil, ganz aus dem Eignen, steht mir zuoberst im ganzen Lebenswerk. Was aber Einsamkeit heißt, Vereisung, wer weiß es wenn nicht ich, die tote Luft um mich entsaugt mir das Mark, zerspellt den Baum in der Wüstenei." (BaN II, 958) 
Die in Wolfskehls Gedicht mit dem Baum tanzende nackte Windsbraut hat darüber hinaus in der Walpurgisnacht ein weiteres und noch direkteres Vorbild im Tanz Fausts mit der jungen Hexe und in dem Wechselgesang, in dem sie beide den Apfelbaum des Paradieses als Anspielung auf den menschlichen Körper verstehen. Die sexuellen Anspielungen sind da so überdeutlich wie unmittelbar vorher in Mephistos Warnung an Faust vor den Haaren der Lilith.23 Wie der Anfang von "Medico Magistrali" deutlich an die Madrigalverse der Faustschen Monologe erinnerte, so hier das Ende an die der Walpurgisnacht. Doch wird dabei zuletzt am deutlichsten Wolfskehls eigene Stimme vernehmbar; der Sturm als Windsbraut wird ihm zur Riesin und ihr Wettern zu einem tosenden Lied, das er dennoch in befreiender Glückserfahrung von dem Gesang eines, nein, seines Vögleins übertönt hört:

Und unsichtbar mein Vöglein, weil sie wettert,

Das Lied der Riesin siegreich überschmettert:

Sein Herz pocht machtvoll, ob es jauchzt, ob bangt, Denn es ist keines, das im Nihil hangt.

Der Bezug zur Walpurgisnacht und zum Ende von Faust II ist da durchaus gewahrt - und die Stimmung zugleich in eine strahlende, ganz und gar untragische Tonart transponiert. In der "Walpurgisnacht"-Szene heißt es, als die Baumstämme krachend übereinanderstürzen: "Aufgescheucht fliegen die Eulen." (V. 3942) Natürlich ist die Eule, und genauer der Kauz, nach altem Volksglauben der Totenvogel, der mit seinem Ruf den nahenden Tod ankündigt. Dieser Vogel begegnet deshalb zuletzt auch dem uralten, vereinsamten Faust, ohne daß der seine Bedeutung erkennen will oder kann, kurz vor seinem Tod in der Szene "Mitternacht". Faust reagiert mit den Versen:

Ein Vogel krächzt; was krächzt er? Mißgeschick.

Von Aberglauben früh und spat umgarnt:

Es eignet sich, es zeigt sich an, es warnt.

Und so verschüchtert, stehen wir allein.

23 Die Haare der Lilith sind natürlich das weibliche Pendant zu den Haaren, an die Simsons Sexualkraft gebunden ist. - Gleichsetzung von Haaren und Blättern (und also von Mensch und Baum) findet sich im Dritten Akt von Faust II in der Szene "Schattiger Hain" (V. 9992-9995), doch stehen diese Verse in Rhythmus und Wortwahl dem Gedicht Wolfskehls ganz fern. 
Die Pforte knarrt, und niemand kommt herein.

(V. 11412-11419)

Durch die "knarrende" Pforte, die dem krächzenden Vogel korrespondiert und wiederum an die knarrende Schublade in Wolfskehls Gedicht erinnert, kommt, ohne daß Faust sie bemerkt, die Sorge herein, um ihn mit Blindheit anzuhauchen, so daß er auch den nahenden Tod nicht bemerkt und sogar noch die Bedeutung der Grabgeräusche für sein eigenes Grab verkennt. Statt dem Totenvogel des alten Faust begegnet das Ich in Wolfskehls Gedicht jedoch seinem bis zur Unsichtbarkeit unscheinbaren Vöglein. Dessen Gesang übertönt laut und deutlich die Stürme des Winters, in dem Wolfskehl nicht nur von der eisigen Kälte der Jahreszeit und der Zeiten, sondern auch von einer Herzattacke niedergeworfen wurde, und das Lied des Vögleins hilft dem Kranken wieder auf, weil er aus diesem Lied statt "Mißgeschick" und Tod eine entgegengesetzte, lebenverheißende Botschaft heraushört.

Damit bestätigt sich im Schlußsextett des Gedichts die Fruchtbarkeit der dialogischen Form, wie sie Wolfskehl, Jean Pauls Unterscheidung von Sprech- und Schreibmenschen zitierend und sich selbst als Sprechmenschen bezeichnend, in einem Brief an Salin vom 17. 12. 1940 für sein "Sprechen" reklamiert hatte. Empfangene Briefe, schreibt er da, seien ihm Anlaß zu "Spruch und Gegenspruch, welch eine Wünschelrute für den Sprung einer Quelle" (BaN II, 141). "Medico Magistrali" beginnt in den ersten beiden Versen mit einem Spruch, in dem der Dichter die telefonische Ferndiagnose dès Dr. Grièsbāch aufgenommen haben dürfte, den Verfallsprozeß des Alterns als schicksalhafte Notwendigkeit akzeptierend: ein Spruch, den er an immer wieder neuen Beispielen bestätigt um schließlich "doch" mit einem Gegenspruch zu enden. In ihm erscheint der vorige Spruch ebenso übertönt wie vom Lied des Vögleins der Sturm der Natur, der dem Dichter symbolisch für den Sturm der Menschheit steht, von dem sein persönliches Schicksal ein Teil ist. Daß die eigene Quelle hier tatsächlich wieder kräftig "springt" - und nicht, wie in Mephistos Bild des Alterns, nur "trübe fließt" - ist ihm Beleg dafür, daß das Schicksal ihm in diesem einen, ihm wichtigsten Punkt trotz allem gewogen blieb: Noch lebt er in seiner sich lebendig erneuernden Dichtung. Im Hauptteil des Gedichts hatte er seine körperlichen Beschwerden und sein Leiden als altersbedingt aus der Mode gekommener Liebhaber24 und Dichter nicht einfach dadurch bannen können, daß er 
sie beim Namen nannte. Der Blick auf den tragisch endenden Faust erlaubte ihm, Abstand zum eigenen Leiden zu gewinnen, doch hätte die daraus resultierende Selbstironie im Prinzip auch in bitterem Sarkasmus enden können. Statt dessen verhilft dem Dichter seine Begegnung mit der neuseeländischen Natur zur Neubelebung und Neubeglaubigung seiner Geistes- und Seelenfreiheit. Er begreift sie als Freiheit von der verachteten Nichtigkeit, durch die jede Mode, bis hin zur schlimmsten der damals herrschenden Nazibarbarei, dazu verdammt ist, zum Opfer ihrer selbst zu werden. Bereits in einem Brief an Faber du Faur vom 17. 12. 1940 hatte er mit Bezug auf die Gewaltherrschaft der Nazis die Gewißheit geäußert, daß “das furchtbare Gespenst überblähter seelen- und geistfeindlicher Nichtigkeit von den Strahlen aufdämmernder Freiheitssonne zu schmelzen beginnt!" (BaN II, 86) Der letzte Vers des Gedichts zeigt die Überwindung der Furcht des Dichters nicht nur vor der mörderischen Nazidiktatur, sondern vor dem Diktat jeder durch ihre Nichtigkeit zum Untergang verurteilten Mode.

Daß der letzte Vers hier ohne den geringsten Rest von Ironie und Selbstironie auskommt, ist keinesweg Indiz für ein auf Dauer zurückgewonnenes Selbstbewußtsein. Wie das schwierige Verhältnis zu Thomas Mann belegt, hatte die durch die Dichtung erreichte Selbstheilung immer nur vorläufige Wirkung. Im Kampf gegen die Nichtigkeit der Mode und der Moden blieb Wolfskehl auf immer neue Selbstbestätigung im dichterischen Werk angewiesen, und der gelegentliche Rückgriff auf Ironie und Selbstironie spielte dabei wiederholt eine keineswegs unwichtige Rolle.25

24 Das letztere ergibt sich aus der intertextuellen Beziehung zur Walpurgisnacht, durch die die Sexualsymbolik von Dübel und Schuber ganz offensichtlich ist. Das Gelegenheitsgedicht "Heilig und heidnisch" (GW I, 269) behandelt dieselbe Thematik mit vielfältigen Bezügen zu anderen Bereichen der europäischen Kulturgeschichte, vom griechischen Mythos über das mittelalterliche Epos bis zu Richard Wagner.

25 Den Zusammenhang von Wolfskehls in Neuseeland verfaßter Gelegenheitsdichtung mit seinem religiösen Spätwerk behandele ich in meinem Vortrag "»Zurufe von fernher«. Karl Wolfskehl in Neuseeland" im Tübinger Karl Wolfskehl Symposion 1998. (Die Beiträge des Tübinger Symposions erscheinen im September 1999 im Stauffenburg Verlag Tübingen, hersg. von Paul Hoffmann unter Mitarbeit von Klaus Bruckinger.) 